\title{
Neumonía varicelosa en adultos: 30 casos
}

\author{
J. C. RODRÍGUEZ BORREGÁN, M. J. DOMÍNGUEZ ARTIGA, E. MIÑAMBRES, \\ E. TEJERINA ÁLVAREZ, M. S. HOLANDA PEÑA, C. GONZÁLEZ FERNÁNDEZ, \\ A. QUESADA SUESCUN
}

Servicio de Medicina Intensiva. Hospital Universitario Marqués de Valdecilla. Santander

\author{
VARICELLA PNEUMONIA IN ADULTS: 30 CASES
}

\section{RESUMEN}

Objetivos: Estudio retrospectivo de los pacientes >15 años que ingresaron en nuestro hospital por neumonía varicelosa en los últimos 10 años.

Métodos: Se incluyeron 30 pacientes, 16 varones y 14 mujeres. La edad media era de 32,73 \pm 7,67 años (Rango: $15-58$ ). El 90\% eran fumadores y 3 estaban embarazadas. La estancia media en el hospital fue de $14,96 \pm 12,02$ días (Rango: 4-57).

Resultados: Ingresaron en UCI 7 pacientes y 2 necesitaron ventilación mecánica. El infiltrado intersticial (70\%) y el alveolo-intersticial (23\%) fueron los patrones radiológicos más frecuentes. La auscultación pulmonar fue normal en 2/3. Los hallazgos clínicos más frecuentes fueron: fiebre $(100 \%)$, tos seca $(86,6 \%)$, disnea $(66,6 \%)$ y dolor pleurítico $(50 \%)$. El índice $\mathrm{pO}_{2} / \mathrm{FiO}_{2}$ fue $\leq 300$ en 15 casos. El $50 \%$ presentaron hiponatremia y el $40 \%$ trombopenia. Falleció un paciente, otro evolucionó a una fibrosis pulmonar y tres desarrollaron, posteriormente, asma.

Conclusiones: La neumonía varicelosa en adultos suele presentarse en individuos fumadores. Se debe realizar Rx tórax a todos los pacientes adultos con varicela e ingresar las neumonías. El tratamiento de elección es el Aciclovir endovenoso y en enfermos graves puede considerarse la asociación de corticoides.

PALABRAS CLAVE: Neumonía varicelosa. Adulto. Aciclovir. Varicela.

\begin{abstract}
Objectives: During the past 10 years, 30 adults (age $>15$ years) were treated for varicella pneumonia in our centre.

Methods: There were 16 males and 14 females. Ages ranged from 15 to 58 years (mean, $32.73 \pm 7.67$ years). Twenty-seven patients $(90 \%)$ were nonpregnant adult smokers and three patients (10\%) were pregnant women. The hospital stay ranged from 4 to 57 days (mean, 14.96 12.02 days).

Results: Seven patients $(23.3 \%)$ were managed in the intensive care unit and two patients $(6,6 \%)$ required mechanical ventilation.

The most common radiographic findings were interstitial infiltrates in twenty-one patients (70\%) and interstitial-alveolar infiltrates in seven patients $(23.3 \%)$. Physical examination of the chest did not reveal abnormalities in twenty patients $(66,6 \%)$. Fifteen patients (50\%) were severely hipoxic with $\mathrm{pO} / \mathrm{FiO}$ ratio less than 300 . Twelve patients (40\%) presented thrombocytopenia and fifteen (50\%) presented hyponatremia. The most frequent clinical features included: fever (100\%), dry cough $(86.6 \%)$, dysnea (66.6\%) and chest pain (50\%). One patient (3.3\%) died. Three patients (10\%) developed asthma and one other patient developed pulmonary fibrosis.

Conclusions: Smoking is associated with an increase incidence of varicella pneumonia in adults. A chest $x$-ray should be practised in all cases of varicella in adults and they all should also be admitted to hospital. Intravenous aciclovir is recommended for treatment of varicella pneumonia in adults and in seriously ill patients the association of corticosteroids should be considered.
\end{abstract}

KEY WORDS: Varicella pneumonia. Adult. Acyclovir. Chickenpox.

Rodríguez Borregán JC, Domínguez Artiga MJ, Miñambres E, Tejerina Álvarez E, Holanda Peña MS, González Fernández, C, Quesada Suescun A. Neumonía varicelosa en adultos: 30 casos. An Med Interna (Madrid) 2003; 20: 612-616.

\section{INTRODUCCIÓN}

La varicela, producida por la primoinfección del virus varicela-zóster (VZV), es la enfermedad exantemática más frecuente en la población infantil de los países desarrollados (1). Se caracteriza por fiebre, malestar general, cefalea y un típico exantema en distintos estadios evolutivos, con menor afectación de partes distales de extremidades y que puede afectar mucosas. Es considerada, excepto en la primera semana de vida, una enfermedad benigna y sus principales complicaciones son neurológicas y sobreinfecciones.

Afecta solamente al 1-2\% de los adultos (2). Representando este segmento de población menos del $5 \%$ de las varicelas pero más de un tercio de los fallecidos (3). La complicación más frecuente (15\%) y grave, en este grupo de edad, es la neumonía varicelosa, ocurriendo el $90 \%$ de ellas en mayores de 19 años (3).

Trabajo aceptado: 19 de mayo de 2003

Correspondencia: Juan Carlos Rodríguez Borregán. Servicio de Medicina Intensiva. Hospital Universitario Marqués de Valdecilla. Avda. Valdecilla, s/n. 39008 Santander, Cantabria. 


\section{MATERIAL Y MÉTODOS}

Estudio retrospectivo de los pacientes mayores de 15 años que ingresaron en nuestro hospital por neumonía varicelosa entre el 1 de enero de 1992 y el 31 de diciembre de 2001. El diagnóstico se basó en criterios clinicorradiológicos en el curso de una varicela. Recogimos los siguientes datos: edad, sexo, fecha de ingreso, días de estancia en el hospital y en la UCI, días de ventilación mecánica, días desde la aparición del rash hasta el ingreso en el hospital, antecedentes personales y contacto reciente con la enfermedad, radiología, clínica, auscultación pulmonar, analítica plasmática, complicaciones, resultados microbiológicos, tratamiento y evolución.

\section{RESULTADOS}

En los 30 casos encontrados, 16 varones y 14 mujeres, la edad media era de $32,73 \pm 7,67$ años (rango entre 15 y 58), siendo por sexos $33,25 \pm 9,65$ en los varones y $32,14 \pm 4,81$ en las mujeres. La incidencia fue de 3 casos/año, destacando los años 2001 y 1993 con 8 y 6 casos. El 53,3\% se presentaron en los meses fríos (diciembre-marzo) y el $30 \%$ en los cálidos (mayo-septiembre).

Como antecedentes personales (Tabla I), 27 eran fumadores y uno exfumador (desde hacía dos años), 2 eran usuarios de drogas por vía parenteral (UDVP) y 3 ex-UDVP (dos estaban infectados por el VIH), 2 sufrían diabetes mellitus, 2 tomaban anticonceptivos orales, un paciente se encontraba en tratamiento con esteroides tópicos por rinitis alérgica y otro era EPOC (bronquitis crónica). Tres pacientes estaban embarazadas, de 5 y 19 semanas que se sometieron, posteriormente, a una interrupción voluntaria del embarazo (IVE) y otra de 36 semanas a la que se realizó una cesárea el $10^{\circ}$ día de ingreso, por amniorrexis espontánea, con feto normal.

El contacto previo con la varicela fue reconocido por el $63,33 \%$, todos entre 1 y 4 semanas. El periodo desde la aparición del exantema hasta el ingreso hospitalario fue de 4,63 $\pm 3,21$ días (rango de 1 a 14), siendo en el 83,3\% entre 2 y 6 días.

La estancia media en el hospital fue de 14,96 \pm 12,02 días (rango: 4-57) y en la UCI, donde ingresaron 7 pacientes $(23,33 \%), 11,28 \pm 14,06$ días. Sólo $2(6,67 \%)$ necesitaron ventilación mecánica (12 y 23 días).

Los patrones radiológicos encontrados fueron: infiltrado intersticial en 21, infiltrado alveolo-intersticial bilateral en 7 y condensación alveolar en 2 (en uno de ellos asociado con infiltrado intersticial). La auscultación pulmonar fue: normal en $20(66,6 \%)$, crepitantes en $6(20 \%)$, sibilancias en $2(6,6 \%)$ $\mathrm{y}$ asociadas a roncus en otros $2(6,6 \%)$.

El índice $\mathrm{pO}_{2} / \mathrm{FiO}_{2}$ fue $\leq 200$ en 4 casos $(13,33 \%)$, entre 201 y 300 en $11(36,67 \%)$ y >300 en 15 (50\%). Los síntomas más frecuentes fueron: fiebre $(100 \%)$, que en el $60 \%$ era $\geq$ $39^{\circ} \mathrm{C}$, tos seca $(86,6 \%)$, disnea $(66,6 \%)$ y dolor pleurítico $(50 \%)$ (Tabla I).

La hiponatremia $(50 \%)$, aunque sólo en dos casos fue menor de $130 \mathrm{mEq} / \mathrm{L}$, trombopenia (40\%) y aumento de LDH (10/17) y AST/ALT (12/17), fueron las principales alteraciones en la analítica plasmática.

Entre las complicaciones (Tabla II) destacar: trombosis venosa profunda en 2 pacientes (uno desarrolló un tromboembolismo pulmonar) y flebitis por la vía endovenosa en otros 2, y en un caso, cada una, distress respiratorio del adulto (SDRA), neumotórax espontáneo, insuficiencia renal (creatinina máxima de $2 \mathrm{mg} / \mathrm{dl}$ ) y hepatitis leve (hepatomegalia, dolor abdominal y aumento de transaminasas) que se resolvió al retirar el aciclovir. Tres pacientes sufrieron sobreinfección bacteriana: neumonía por Candida parapsilosis, neumonía por neumococo en otro paciente y sobreinfección por neumococo, Escherichia coli y Acinetobacter baumanni en otro.

Todos, excepto uno, recibieron aciclovir, la mitad en forma endovenosa durante 7-10 días y el resto con pautas muy variadas (Tabla II). Fue instaurado tratamiento antibiótico empírico precoz en 6 casos.

Falleció una paciente (infectada por el VIH), tras una parada cardíaca inesperada, el $4^{\circ}$ día de ingreso. Tres pacientes, posteriormente, desarrollaron asma y el SDRA evolucionó a una fibrosis pulmonar. En el resto de los pacientes la evolución fue buena. Dos pacientes (que acudieron al hospital por otro motivo) seguían presentando, 6 años después, nódulos pulmonares calcificados, en la radiografía de tórax.

\section{DISCUSIÓN}

La inmunodepresión, enfermedades malignas, tratamiento con citotóxicos o esteroides, edad avanzada, EPOC, embarazo (sobre todo en el 3er trimestre), tabaquismo y el sexo masculino son considerados los principales factores de riesgo $(4,5)$. En nuestra serie apreciamos una alta incidencia en relación con el tabaco, como ya es apuntada por otros autores $(5,6)$. No hemos encontrado diferencias por sexo. Sólo un paciente tenía una EPOC y la mayor frecuencia observada en los meses fríos no se corresponde con otras series de nuestro medio $(6,7)$.

La varicela es altamente contagiosa, de forma directa (gotitas de Pflügge) y por contacto con las lesiones cutáneas, desde 48 horas antes hasta 4-5 días después de aparecer el exantema y su periodo de incubación es de unos 15 días (11 días a 1 mes).

La sintomatología de la neumonía varicelosa consiste en: fiebre, tos seca y disnea (los más frecuentes), dolor pleurítico y más raro hemoptisis. Los síntomas respiratorios, aunque pueden precederle en 24 horas, normalmente aparecen de 1 a 6 días tras el rash (8). Suele existir disociación clínico-radiológica con una auscultación pulmonar anodina (9).

El patrón radiológico más frecuente es el infiltrado intersticial bilateral, con nódulos de 2 a $10 \mathrm{~mm}$ (10), con predominio en bases y/o región perihiliar, aunque no es raro el patrón alveolar, estas lesiones pueden tardar meses en desaparecer o evolucionar hacia la calcificación residual, estando discutida su evolución hacia neumonitis intersticial usual. El derrame pleural y las adenopatías mediastínicas son poco frecuentes $(6,11)$.

El diagnóstico habitualmente se realiza por criterios clinicorradiológicos en el curso de una varicela, siendo la serología, el cultivo y el frotis de Tzanck pruebas confirmatorias. En nuestro caso se confirmó con serología en 19 pacientes.

Las alteraciones analíticas más frecuentes son la trombopenia transitoria (inicialmente por acción directa del virus o más tardía por anticuerpos antiplaquetas), la hiponatremia leve y una elevación moderada de la AST, ALT, LDH y fosfatasa alcalina.

El tratamiento de elección es el aciclovir endovenoso (10-15 $\mathrm{mg} / \mathrm{kg} / 8 \mathrm{~h}$ ), especialmente en inmunocomprometidos, embarazadas y en casos graves. En pacientes con buen estado general y 
TABLA I

DATOS EPIDEMIOLÓGICOS, RADIOLÓGICOS, DE AUSCULTACIÓN PULMONAR Y GASOMÉTRICOS

\begin{tabular}{|c|c|c|c|c|c|c|c|}
\hline$\overline{N^{0}}$ & Edad/Sexo & $\begin{array}{l}\text { Día UCI/ } \\
\text { hospital }\end{array}$ & $\begin{array}{l}\text { Contacto previo } \\
\text { con varicela }\end{array}$ & Antecedentes & Patrón radiológico & $\begin{array}{l}\text { Auscultación } \\
\text { pulmonar }\end{array}$ & $\begin{array}{l}D(A-a) \\
02\end{array}$ \\
\hline 1 & $31 / M$ & $6 / 15$ & $2 \mathrm{sem}$ & 40 cig/día & Alveolo-intersticial & Normal & 90 \\
\hline 2 & $37 / \mathrm{V}$ & $0 / 22$ & $1 \mathrm{sem}$ & 40 cig/día & Alveolo-intersticial & Normal & 180 \\
\hline 3 & $32 / M$ & $0 / 14$ & $3 \mathrm{sem}$ & 20 cig/día & $\begin{array}{l}\text { Intersticial y condensación } \\
\text { alveolar en base derecha }\end{array}$ & Normal & 60 \\
\hline 4 & $30 / M$ & $0 / 14$ & $2 \mathrm{sem}$ & $\begin{array}{l}\text { Embarazada (19 sem), } \\
10 \text { cig/día }\end{array}$ & Intersticial & Normal & 70 \\
\hline 5 & $37 / \mathrm{V}$ & $4 / 14$ & $2 \mathrm{sem}$ & 30 cig/día & Intersticial & $\begin{array}{l}\text { Sibilancias } \\
\text { y roncus }\end{array}$ & 160 \\
\hline 6 & $33 / M$ & $0 / 11$ & $1 \mathrm{sem}$ & 40 cig/día & Intersticial & Normal & 90 \\
\hline 7 & $15 / \mathrm{V}$ & $0 / 9$ & & En tto con esteroides & Alveolo-intersticial & Normal & 70 \\
\hline 8 & $33 / \mathrm{V}$ & $2 / 9$ & & 20 cig/día & Intersticial & Normal & 160 \\
\hline 9 & $36 / \mathrm{V}$ & $0 / 7$ & $2 \mathrm{sem}$ & 30 cig/día & Alveolo-intersticial & Normal & 230 \\
\hline 10 & $39 / \mathrm{V}$ & $3 / 14$ & & Síndrome de Down & Alveolo-intersticial & $\begin{array}{l}\text { Sibilancias } \\
\text { y roncus }\end{array}$ & 85 \\
\hline 11 & $38 / M$ & $0 / 11$ & $2 \mathrm{sem}$ & 20 cig/día. & Alveolo-intersticial & Crepitantes & 80 \\
\hline 12 & $28 / M$ & $3 / 11$ & & DM, 10 cig/día & Intersticial & Normal & 95 \\
\hline 13 & $32 / M$ & $39 / 56$ & $2 \mathrm{sem}$ & $\begin{array}{l}\text { Embarazada (36 sem), } \\
10 \text { cig/día }\end{array}$ & Intersticial & Normal & 580 \\
\hline 14 & $30 / \mathrm{V}$ & $0 / 10$ & $1 \mathrm{sem}$ & 20 cig/día & Intersticial & Crepitantes & 140 \\
\hline 15 & $24 / \mathrm{V}$ & $0 / 14$ & & 20 cig/día & Intersticial & Normal & 200 \\
\hline 16 & $31 / M$ & $0 / 12$ & 4 sem & 20 cig/día & Intersticial & Normal & 75 \\
\hline 17 & $29 / \mathrm{V}$ & $0 / 6$ & 3 sem & 20 cig/día & Condensación alveolar & Normal & 60 \\
\hline 18 & $27 / \mathrm{V}$ & $0 / 19$ & & 20 cig/día & Intersticial & Normal & 40 \\
\hline 19 & $33 / M$ & $0 / 16$ & $2 \mathrm{sem}$ & ACO, 20 cig/día & $\begin{array}{l}\text { Intersticial (persistía } \\
3 \text { años después) }\end{array}$ & Normal & 75 \\
\hline 20 & $29 / \mathrm{V}$ & $0 / 9$ & & UDVP, 20 cig/día & Intersticial & Normal & 70 \\
\hline 21 & $24 / M$ & $0 / 8$ & $2 \mathrm{sem}$ & Ex-UDVP, 40 cig/día & Intersticial & Normal & 75 \\
\hline 22 & $40 / \mathrm{V}$ & $0 / 18$ & 3 sem & 20 cig/día & Intersticial & Sibilancias & 60 \\
\hline 23 & $30 / M$ & $22 / 57$ & & ACO, 20 cig/día & Intersticial & Normal & 90 \\
\hline 24 & $34 / \mathrm{V}$ & $0 / 4$ & 4 sem & $\begin{array}{l}20 \text { cig/día, ex-UDVP, } \\
\text { VHC + }\end{array}$ & Intersticial & Normal & 60 \\
\hline 25 & $41 / \mathrm{V}$ & $0 / 16$ & $3 \mathrm{sem}$ & 20 cig/día & Intersticial & Crepitantes & 70 \\
\hline 26 & $39 / M$ & $0 / 14$ & $3 \mathrm{sem}$ & $\begin{array}{l}\text { Embarazada (5 sem), } \\
\text { exfumadora (2 años) }\end{array}$ & Intersticial & Normal & 60 \\
\hline 27 & $42 / M$ & $0 / 8$ & 4 sem & 30 cig/día. & Intersticial & Crepitantes & 70 \\
\hline 28 & $27 / M$ & $0 / 5$ & & $\begin{array}{l}20 \mathrm{cig} / \text { día, ex-UDVP, } \\
\text { VIH+, VHC+ y VHB + }\end{array}$ & Intersticial & Crepitantes & 80 \\
\hline 29 & $58 / \mathrm{V}$ & $0 / 12$ & & $\begin{array}{l}20 \text { cig/día, bronquitis } \\
\text { crónica, DM II }\end{array}$ & Alveolo-intersticial & Sibilancias & 70 \\
\hline 30 & $23 / \mathrm{V}$ & $0 / 14$ & & $\begin{array}{l}40 \text { cig/día, UDVP, VIH+, } \\
\text { VHC+, VHB+ }\end{array}$ & Intersticial & Crepitantes & 60 \\
\hline
\end{tabular}

UDVP= usuario de drogas por vía parenteral; $A C O=$ anticonceptivos orales; $D M=$ diabetes mellitus.

escasa repercusión de la ventilación se ha ensayado, con buen resultado, por vía oral $(12,13)$. El aciclovir puede producir flebitis, molestias gastrointestinales, trastornos neurológicos y nefropatía obstructiva reversible (la infusión lenta y la hidratación minimizan este riesgo) (5). En nuestra serie la insuficiencia renal, la hepatitis y las flebitis, fueron atribuidas a este fármaco. En pacientes graves quizás los corticoides puedan mejorar el pronóstico $(3,7)$. No parece justificado el uso de antibioterapia empírica precoz y en nuestros pacientes las sobreinfecciones bacterianas fueron tardías. 
TABLA II

CLÍNICA, COMPLICACIONES, TRATAMIENTO Y EVOLUCIÓN

\begin{tabular}{|c|c|c|c|c|}
\hline$\overline{N^{0}}$ & Clínica & Complicaciones & Días con Aciclovir & Evolución \\
\hline 1 & $\begin{array}{l}\text { Tos seca, dolor torácico, disnea, } \\
\text { taquipnea, mialgias, fiebre }\left(40^{\circ} \mathrm{C}\right)\end{array}$ & $\begin{array}{l}\text { Trombopenia, hiponatremia, } \\
\uparrow \text { ALT/AST }\end{array}$ & $13 \mathrm{ev}$ & Buena \\
\hline 2 & Tos seca, dolor torácico, disnea, taquipnea, fiebre $\left(40^{\circ} \mathrm{C}\right)$ & Trombopenia, $\uparrow$ ALT/AST & $8 \mathrm{ev}$ & Buena \\
\hline 3 & Tos seca, disnea, mialgias, odinofagia, fiebre $\left(38^{\circ} \mathrm{C}\right)$ & Trombopenia & $6 \mathrm{ev}$ & Buena \\
\hline 4 & Tos seca, dolor torácico, disnea, taquipnea, fiebre $\left(39^{\circ} \mathrm{C}\right)$ & IVE & 5 ev y 5 vo & Buena \\
\hline 5 & $\begin{array}{l}\text { Tos seca, dolor torácico, disnea, hemoptisis, } \\
\text { hepatomegalia, fiebre }\left(38^{\circ} \mathrm{C}\right)\end{array}$ & $\begin{array}{l}\text { Trombopenia, hiponatremia, } \\
\uparrow A L T / A S T\end{array}$ & $10 \mathrm{ev}$ & Buena \\
\hline 6 & Tos seca, dolor torácico, disnea, fiebre $\left(38^{\circ} \mathrm{C}\right)$ & Hiponatremia. & 4 ev y 4 vo & Buena \\
\hline 7 & Tos seca, adenopatías, fiebre $\left(38^{\circ} \mathrm{C}\right)$ & Hiponatremia. & $7 \mathrm{ev}$ & Buena \\
\hline 8 & Tos seca, dolor torácico, odinofagia, fiebre $\left(39^{\circ} \mathrm{C}\right)$ & Hiponatremia. & $10 \mathrm{ev}$ & Buena \\
\hline 9 & Tos seca, fiebre $\left(40^{\circ} \mathrm{C}\right)$ & $\begin{array}{l}\text { Trombopenia, hiponatremia, } \\
\uparrow \text { ALT/AST }\end{array}$ & $5 \mathrm{ev}$ & Buena \\
\hline 10 & Tos seca, dolor torácico, disnea, fiebre $\left(40^{\circ} \mathrm{C}\right)$ & Flebitis, hiponatremia & 4 ev y 8 vo & Buena \\
\hline 11 & Dolor torácico, disnea, fiebre $\left(40^{\circ} \mathrm{C}\right)$ & $\begin{array}{l}\text { Insuficiencia renal, trombopenia, } \\
\uparrow \text { ALT/AST }\end{array}$ & $7 \mathrm{ev}$ & Buena \\
\hline 12 & Tos seca, vómitos, cefalea, taquipnea, fiebre $\left(39^{\circ} \mathrm{C}\right)$ & & $8 \mathrm{ev}$ & $\begin{array}{l}\text { Nódulos } \\
\text { pulmonares } \\
\text { calcificados } \\
6 \text { años } \\
\text { después }\end{array}$ \\
\hline 13 & Tos seca, dolor torácico, disnea, fiebre $\left(39^{\circ} \mathrm{C}\right)$ & $\begin{array}{l}\text { SDRA, neumonía por Candida } \\
\text { parapsilosis, cesárea con feto normal }\end{array}$ & $28 \mathrm{ev}$ & Buena \\
\hline 14 & Tos seca, dolor torácico, disnea, adenopatías, fiebre $\left(40^{\circ} \mathrm{C}\right)$ & Hiponatremia & 7 ev y 5 vo & Buena \\
\hline 15 & Tos seca, disnea, odinofagia, adenopatías, fiebre $\left(39^{\circ} \mathrm{C}\right)$ & $\begin{array}{l}\text { Trombopenia, hiponatremia hepatitis, } \\
\uparrow \text { ALT/AST }\end{array}$ & $9 \mathrm{ev}$ & Asma \\
\hline 16 & Tos seca, odinofagia, artromialgias, fiebre $\left(38^{\circ} \mathrm{C}\right)$ & $\uparrow \mathrm{ALT} / \mathrm{AST}$ & 10 ev y 4 vo & Asma \\
\hline 17 & Tos seca, dolor torácico, cefalea, odinofagia, fiebre $\left(40^{\circ} \mathrm{C}\right)$ & & $\sin$ tto & Buena \\
\hline 18 & Adenopatías submaxilares, fiebre $\left(39^{\circ} \mathrm{C}\right)$ & $\begin{array}{l}\text { Trombopenia, hiponatremia, } \\
\uparrow \text { ALT/AST }\end{array}$ & 12 vo & $\begin{array}{l}\text { Asma y } \\
\text { nódulos } \\
\text { pulmonares } \\
\text { calcificados } \\
\text { años } \\
\text { después }\end{array}$ \\
\hline 19 & Dolor torácico, disnea, adenopatías cervicales, fiebre $\left(39^{\circ} \mathrm{C}\right)$ & TVP y TEP, $\uparrow$ ALT/AST & $15 \mathrm{ev}$ & Buena \\
\hline 20 & Tos seca, dolor torácico, disnea, fiebre $\left(38^{\circ} \mathrm{C}\right)$ & Trombopenia, hiponatremia. & $7 \mathrm{ev}$ & Buena \\
\hline 21 & Tos seca, disnea, fiebre $\left(40^{\circ} \mathrm{C}\right)$ & Hiponatremia & 7 ev y 8 vo & Buena \\
\hline 22 & Tos seca, odinofagia, fiebre $\left(40^{\circ} \mathrm{C}\right)$ & $\begin{array}{l}\uparrow \text { ALT/AST, TVP, flebitis, } \\
\text { sobreinfección por neumococo }\end{array}$ & $10 \mathrm{ev}$ & Buena \\
\hline 23 & Tos seca, dolor torácico, disnea, fiebre $\left(38^{\circ} \mathrm{C}\right)$ & $\begin{array}{l}\text { Trombopenia, hipoalbuminemia, } \\
\text { neumotórax, sobreinfección } \\
\text { por neumococo, E. coli, A. baumanni }\end{array}$ & $8 \mathrm{ev}$ & $\begin{array}{l}\text { Fibrosis } \\
\text { pulmonar }\end{array}$ \\
\hline 24 & Fiebre $\left(38,6^{\circ} \mathrm{C}\right)$ & $\uparrow \mathrm{ALT} / \mathrm{AST}, \uparrow \mathrm{LDH}$, hiponatremia & $4 \mathrm{ev}$ & Buena \\
\hline 25 & Expectoración blanquecina, disnea, fiebre $\left(39^{\circ} \mathrm{C}\right)$ & $\begin{array}{l}\text { Trombopenia, } \uparrow \text { ALT/AST, } \\
\uparrow \mathrm{LDH} \text {, hiponatremia }\end{array}$ & 9 ev y 2 vo & Buena \\
\hline 26 & Tos seca, disnea, fiebre $\left(38^{\circ} \mathrm{C}\right)$ & $\uparrow \mathrm{LDH}, \mathrm{IVE}$ & 8 ev y 3 vo & Buena. \\
\hline 27 & Tos seca, disnea, fiebre $\left(39^{\circ} \mathrm{C}\right)$ & Leucocitosis & $7 \mathrm{ev}$ & Buena \\
\hline 28 & $\begin{array}{l}\text { Tos, expectoración herrumbrosa, dolor torácico, } \\
\text { disnea, fiebre }\left(38^{\circ} \mathrm{C}\right)\end{array}$ & Leucopenia & $8 \mathrm{ev}$ & Exitus \\
\hline 29 & Tos seca, disnea, fiebre $\left(38,5^{\circ} \mathrm{C}\right)$, hemoptisis & Trombopenia, hiponatremia & $7 \mathrm{ev}$ & Buena \\
\hline 30 & Tos, fiebre $\left(38^{\circ} \mathrm{C}\right)$ & Leucopenia, $\uparrow$ LDH & $10 \mathrm{ev}$ & Buena \\
\hline
\end{tabular}

ev= endovenoso; vo= vía oral; SDRA= síndrome de distress respiratorio del adulto; $\mathrm{ALT}=$ alanina aminotransferasa; $\mathrm{AST}=$ aspartato aminotransferasa; $\mathrm{LDH}=$ lactato deshidrogenasa; TVP= trombosis venosa profunda; $\mathrm{TEP}=$ tromboembolismo pulmonar; $\mathrm{PCR}=$ parada cardiorrespiratoria. 
La mejoría suele iniciarse con la resolución del exantema, aunque la evolución es difícil de preveer. La hipoxemia grave temprana (principal indicador de una mala evolución) (9), inmunodeprimidos, embarazadas (14), la necesidad de conexión a ventilación mecánica, la sobreinfección bacteriana y el SDRA están relacionados con peor pronóstico.

La mortalidad en el adulto sano es del 10-20\% (6), en inmunodeprimidos y embarazadas asciende al $11-40 \%(14,15)$ y en pacientes que necesitan ventilación mecánica llega al 50\% $(16,17)$. Sin embargo, las series más recientes publicadas en España reflejan una mortalidad menor. Así, García Quintana (18) $(n=13)$, Aleman (11) $(n=25)$ y De la Peña (7) $(n=22)$, no refieren ningún fallecimiento y De la Cruz (6) $(n=13)$, Quintana (9) $(n=6)$ y Blanch (19) $(n=7)$ informan de uno.

\section{CONCLUSIONES}

La neumonía varicelosa en adultos suele presentarse en individuos fumadores.

Se debe realizar Rx tórax a todos los pacientes adultos con varicela e ingresar las neumonías. El tratamiento de elección es el Aciclovir endovenoso (10-15 mg/kg/8 h) y en enfermos graves puede considerarse la asociación de corticoides.

\section{Bibliografía}

1. Moraga-Llop FA, Campins M. Vacuna de la varicela. Una inmunización del niño y del adulto. Enferm Infecc Microbiol Clin 2000; 18 (10): 516-518.

2. Wallace MR, Bowler WA, Murray NB, Brodine SK, Oldfield EC. Treatment of adult varicella with oral Acyclovir. A randomized, placebocontrolled trial. Ann Intern Med 1992; 117: 358-363.

3. Mer M, Richards GA. Corticosteroids in life-threatening varicella pneumonia. Chest 1998; 114: 355-360.

4. Fernández Vázquez E, Soudan A, Espejo Guerrero A, González Vargas F, Cabrera Torres L. Neumonía varicelosa en un adulto sano. Revisión de factores de riesgo y tratamiento. An Med Interna (Madrid) 1994; 11: 187-188.

5. Blanco García JJ, de Miguel Diez J, Hermida Gutiérrez JA. Neumonía varicelosa: complicaciones del tratamiento antiviral. Arch Bronconeumol 1999; 35: 465-466.

6. De la Cruz Morón I, Alfageme Michavila I, Muñoz Lucena F, Ramos P, Rojas JL, García Polo C. Neumonía varicelosa en adultos: revisión de 13 casos. Arch Bronconeumol 1999; 35: 357-359.

7. de la Peña L, Izaguirre D, Aguirrebengoa K, Grande C, Montejo M. Neumonía por varicela en el adulto: estudio de 22 casos. Enferm Infecc Microbiol Clin 2000; 18: 493-495.

8. Schlossberg D, Littman M. Varicella Pneumonia. Arch Intern Med. 1988; 148: 1630-1632.

9. Quintana González JL, Rodríguez Pascual L, Morato Arnáiz A, García Arroyo I, Martín Torre E. Neumonía varicelosa en adultos sanos. A propósito de 6 casos. Arch Bronconeumol 1996; 32: 369-372.
10. Gogos CA, Bassaris HP, Vagenakis AG. Varicella pneumonia in adults A review of pulmonary manifestations, risk factors and treatment. Respiration 1992; 59: 339-343.

11. Aleman Llanso C, García Quintana AM, Alegre Martín J, Recio Iglesias J, Falco Ferrer V, Fernandez de Sevilla Ribosa T. Neumonía varicelosa en el adulto. Revisión de 25 casos. Rev Clin Esp 1997; 197: 690-692.

12. Huncharck M, Klassen H. Acyclovir in Varicella pneumonia in healthy adults. Respiration 1993; 60: 254-255.

13. Gil ME, Arribas JM, López A, González ML. Neumonía por varicela zoster. Tratamiento por vía oral con aciclovir. Enferm Infecc Microbiol Clin 1995; 13: 193-194.

14. Esmonde TF, Herdman G, Anderson G. Chickenpox pneumonia: an association with pregnancy. Thorax 1989; 44: 812-815.

15. Rodrigues J, Niederman MS. Pneumonia complicating pregnancy. Clin Chest Med 1992; 13: 679-691

16. Feldman S. Varicella-zoster virus pneumonitis. Chest 1994; 106 Suppl: 22S-27S.

17. Haake DA, Zakowski PC, Haake DL, Bryson YJ. Early treatment with acyclovir for varicella pneumonia in otherwise healthy adults: retrospective controlled study and review. Rev Infect Dis 1990; 12: 788-98.

18. García Quintana A, Alegre Martín J, Falco V, Fernández de Sevilla T, Martínez Vázquez JM. Neumonía varicelosa en el adulto. Estudio de 13 casos. Rev Clin Esp 1992; 191: 314-316.

19. Blanch Sancho JJ, Tirado Miranda R, Medrano González F. Neumonía varicelosa en el adulto sano. A propósito de 7 casos. An Med Interna (Madrid) 1998; 15: 338 